\title{
Análisis energético de un sistema termoeléctrico para el aprovechamiento del calor residual en un motor Turbofan mediante dinámica de fluidos computacional
}

\section{Energy Analysis of a thermoelectric system for the use of residual heat in a Turbofan engine through computational fluid dynamics}

ROJAS-AVILA, Apolo†, VALLE-HERNÁNDEZ, Julio*, CASTILLO-JIMÉNEZ, Víctor, GALLARDO-VILLAREAL, José Manuel y ALONSO-ALFARO Jorge G.

Universidad Politécnica Metropolitana de Hidalgo, Maestría en Ingeniería Aeroespacial

Universidad Autónoma del Estado de Hidalgo, Escuela Superior de Apan

ID $1^{\text {er }}$ Autor: Apolo, ROJAS-AVILA / ORC ID: 0000-0003-2934-2533, Researcher ID Thomson: O-4655-2018, CVU CONACYT ID: 905177

ID $1^{\text {er }}$ Coautor: Julio, Valle-Hernández / ORC ID: 0000-0001-8957-0066, Researcher ID Thomson: O-7339-2018, CVU CONACYT ID: 210743

ID $2^{\text {do }}$ Coautor: Víctor, Castillo-Jiménez / ORC ID: 0000-0002-5031-0635

ID $3^{\text {er }}$ Coautor: José Manuel, Gallardo-Villareal / ORC ID: 0000-0002-7578-7229, CVU CONACYT ID: 366394

ID $4^{\text {to }}$ Coautor: Jorge G., Alonso-Alfaro

DOI: $10.35429 / J O E S .2019 .21 .6 .1 .7$

Recibido 9 de Octubre, 2019; Aceptado 30 de Diciembre, 2019

\section{Resumen}

En el presente trabajo se muestra el análisis energético de un sistema termoeléctrico acoplado a un motor turbofan, el cual se basa en el modelado matemático de la transferencia de calor del sistema durante el proceso de cogeneración utilizando dinámica de fluidos computacionales (CFD). El aprovechamiento del calor residual de un motor turbofan nos permitiría incrementar su eficiencia lo cual tiene impacto tanto económico (debido al ahorro de combustible) como ambiental (reducción de gases contaminantes). La sección propuesta para la instalación del sistema termoeléctrico es la que recubre la turbina y la sección de tobera del motor turbofan, ya que son secciones en las que se tienen mejores temperaturas de operación, del orden de 600 a 200 ${ }^{\circ} \mathrm{C}$. Como resultados de la simulación en CFD se obtiene la distribución de temperaturas a través del termoeléctrico y la diferencia de potencial eléctrico generado. Con estos resultados se determina la eficiencia global del sistema y la energía eléctrica generada con el calor residual. La energía generada por el sistema termoeléctrico puede ser aprovechada en sistemas secundarios de la aeronave, como el sistema de iluminación, entretenimiento, etc. La importancia del análisis radica en aumentar la eficiencia general del proceso de combustión del motor, para reducir el consumo de combustible y los costos asociados a éste.

Generador termoeléctrico, calor residual, Simulación CFD

\begin{abstract}
This paper shows the energy analysis of a thermoelectric system coupled to a turbofan engine, which is based on the mathematical modeling of the heat transfer of the system during the cogeneration process using fluid dynamics (CFD). The use of waste heat from a turbofan engine would allow us to increase its efficiency which has both economic (due to fuel savings) and environmental (reduction of polluting gases). The proposed section for the installation of the thermoelectric system is the one that covers the turbine and the nozzle section of the turbofan engine, since they are sections in which there are better operating temperatures, in the order of 600 to $200 \mathrm{oC}$. As results of the CFD simulation you get the distribution of temperatures through the thermoelectric and the difference of electrical potential generated. These results determine the overall efficiency of the system and the electrical energy generated by the waste heat. The energy generated by the thermoelectric system can be used in secondary aircraft systems, such as the lighting system, entertainment, etc. The importance of the analysis lies in increasing the overall efficiency of the engine combustion process, to reduce fuel consumption and the costs associated with it.
\end{abstract}

Thermoelectric Generator, residual heat, CFD Simulation

Citación: ROJAS-AVILA, Apolo, VALLE-HERNÁNDEZ, Julio, CASTILLO-JIMÉNEZ, Víctor, GALLARDOVILLAREAL, José Manuel y ALONSO-ALFARO Jorge G. Análisis energético de un sistema termoeléctrico para el aprovechamiento del calor residual en un motor Turbofan mediante dinámica de fluidos computacional. Revista de Sistemas Experimentales. 2019. 6-21: 1-7

\footnotetext{
* Correspondencia del Autor (jvalle@ upmh.edu.mx)

$\uparrow$ Investigador contribuyendo como primer autor.
} 


\section{Introducción}

Debido al incremento del costo en los combustibles derivados del petróleo, aunado al cambio climático por la contaminación derivada de la combustión de los mismos, nos vemos en la necesidad de hacerlos más eficientes, con la finalidad de reducir el consumo de combustible. Los efectos termoeléctricos fueron descubiertos durante la primera mitad del siglo XIX. El efecto Seebeck, descubierto en 1821 por Thomas Johann Seebeck, explica que, si dos metales diferentes están en contacto y son sometidos a diferentes temperaturas, en sus uniones se obtendrá un potencial eléctrico [1], o a la inversa (efecto Peltier, descubierto en 1834). Existe un tercer efecto llamado Efecto Thomson el cual se define como el intercambio de calor en un material homogéneo sometido a una diferencia de temperaturas entre sus extremos a través del cual se hace circular una corriente eléctrica [2].

Los generadores termoeléctricos (TEGs) pueden utilizarse en motores Diésel y escapes de los vehículos como sistemas de cogeneración; lo cual permite reducir el tamaño del alternador que implica reducir el consumo de combustible[3,4]; la Corporación Marítima de Física Aplicada de Baltimore instalo en 2013 un TEG para producir energía eléctrica del fondo marino aprovechando la diferencia de temperatura entre el agua fría del mar y los fluidos calientes liberados por depósitos geotérmicos; aprovechar el calor del cuerpo para cargar dispositivos móviles [3]; en sondas como la Voyager de la NASA, los generadores termoeléctricos han estado en uso por más de 30 años sin necesidad de mantenimiento; en helicópteros de tobera cónica [5]; así como muchas otras potenciales aplicaciones en el sector aeronáutico y aeroespacial $[6,7,8]$.

Los TEGs son útiles en aplicaciones de cogeneración, por ello en el presente trabajo se propone la implementación de un sistema termoeléctrico, en secciones específicas de un motor turbofan, con la finalidad de aprovechar parte del calor residual, del motor, para la generación de energía eléctrica.

Las altas temperaturas generadas en secciones del motor turbofan, como la tobera y secciones que recubren las turbinas, pueden ser aprovechadas para la generación de energía eléctrica con un sistema termoeléctrico.
El flujo secundario permite obtener un mayor gradiente de temperaturas en los TEG, debido a la convección forzada, por lo que la sección con mayor potencial para su implementación es la superficie externa de la tobera y la sección que recubre las turbinas. Es importante que se tenga el mayor gradiente de temperaturas en las caras del termoeléctrico debido a que es directamente proporcional al potencial eléctrico generado.

En el presente trabajo se realiza el análisis del aprovechamiento del calor residual para la generación de energía eléctrica, a partir del calor residual de un motor turbofan, así como su implementación en sistemas de una aeronave. Esto permite disminuir el consumo de combustible ya que la energía eléctrica consumida por la aeronave proviene de sangrados del motor de propulsión o del sistema APU, que en la mayoría de los casos utiliza el mismo tipo de combustible que los motores propulsores. Al reducir el consumo de combustible se reducen los costos relacionados, además de reducir las emisiones contaminantes.

Entre las potenciales aplicaciones, de TEGs, en el sector aeronáutico y aeroespacial se encuentra el aprovechamiento del calor residual, por lo que en este trabajo se presenta una alternativa para la generación de energía eléctrica a partir del aprovechamiento del calor de los gases de escape de los motores de la aeronave.

\section{Metodología}

Para el presente trabajo se llevó a cabo la siguiente metodología:

1. Elección del motor turbofan para su análisis termodinámico.

2. Selección de la sección del motor en la que se instalaran los generadores termoeléctricos.

3. Modelado de la transferencia de calor de la sección del motor turbofan acoplado al generador termoeléctrico.

4. Estimación de la energía térmica aprovechable por el sistema termoeléctrico.

5. Calculo de la energía eléctrica generada por el sistema termoeléctrico.

6. Determinar los sistemas de la aeronave en los cuales puede ser aprovechada la energía generada. 
7. Resultados y discusión.

\section{Características del motor turbofan}

A partir de los datos del motor Trent 900, que se muestran en la tabla 1, se determinaron las condiciones de operación en cada punto del ciclo de propulsión para el motor Trent 900.

\begin{tabular}{|l|r|}
\hline Longitud & $4.93 \mathrm{~m}$ \\
\hline Diámetro del fan & $2.94 \mathrm{~m}$ \\
\hline Bypass Ratio & 8.4 \\
\hline Mac de vuelo & 0.83 \\
\hline Relación general de presión & 39 \\
\hline Compresor intermedio & 8 etapas \\
\hline Compresor de alta & 6 etapas \\
\hline Turbina de alta presión & 1 etapa \\
\hline Turbina de baja presión & 5 etapas \\
\hline Empuje referencia (vuelo & $1800 \mathrm{~K}$ \\
\hline Temperatura máxima (Tmax $)$ & $11,000 \mathrm{~m}$ \\
\hline $\begin{array}{l}\text { Altura de racera } \\
\text { crucero) }\end{array}$ & $900 \mathrm{~km} / \mathrm{h}$ \\
\hline Velocidad de crucero & $72,000 \mathrm{lbf}$ \\
\hline
\end{tabular}

Tabla 1 Datos del motor Trent 900 de Rolls Royce Fuente: www.rolls-royce.com

La figura 1 muestra el diagrama general de un motor turbofan.

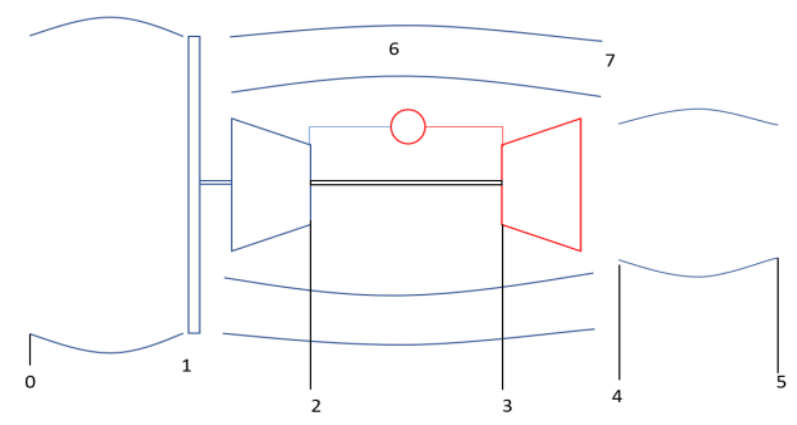

Figura 1 Diagrama de un motor turbofan

Cada uno de los puntos en la figura nos representa las entradas o salidas de flujos, Identificados de la siguiente manera:

0.- Entrada de aire al difusor.

1.- Entrada de aire al compresor, pasando por el fan.

2.- Salida de aire del compresor y entrada a la cámara de combustión.

3.- Salida de gases de la cámara de combustión y entrada a la sección de turbinas.

4.- Salida de gases de combustión de las turbinas y entrada a la tobera.

5.- Salida de gases de combustión de la tobera.

6.- Flujo de aire del flujo secundario.

7.- Salida de aire del flujo secundario.

\section{Simulación CFD de la sección de interés del motor turbofan}

A partir de la temperatura máxima y los flujos, primario y secundario, se realiza una simulación CFD de la sección de interés para determinar la distribución de temperaturas. Las secciones elegidas, para la colocación del sistema termoeléctrico, son la sección que recubre la turbina y la sección de la tobera, debido a que ahí se encuentran temperaturas adecuadas, además de no tener contacto con los gases de escape de la cámara de combustión, y son refrigeradas por el flujo secundario del motor.

En estas secciones se tiene un área disponible de alrededor de $8 \mathrm{~m}^{2}$ para la colocación de termoeléctricos, así como temperaturas que van desde los 200 hasta los 600 ${ }^{\circ} \mathrm{C}$. Para la elaboración del modelo se tomó como temperatura de flujo primario, a la entrada de la cámara de combustión, una temperatura de alrededor de $620 \mathrm{~K}$. A partir de la distribución de temperaturas obtenida se realiza el modelado del sistema termoeléctrico, para determinar el potencial eléctrico generado a partir de la diferencia de temperaturas.

\section{Modelado del sistema termoeléctrico}

En el efecto Seebeck el voltaje producido es proporcional a la diferencia de temperaturas entre las uniones. Esto se relaciona mediante la constante de proporcionalidad (s) llamada coeficiente de Seebeck. Para el análisis del sistema termoeléctrico se parte del balance de energía, como se muestra en la ecuación 1, para la sección a analizar.

$\dot{Q}_{\text {in }}-\dot{Q}_{\text {out }}-S_{\text {cal }}=0$

Donde:

Qin: Calor suministrado en el área de contacto con el termoeléctrico, calor residual del motor.

Qout: Calor que se emite al ambiente que rodea al termoeléctrico (convección radiación).

$\mathrm{S}_{\text {cal }}$ : Sumidero de calor, el cual representa la energía eléctrica generada por el termoeléctrico.

Para determinar la diferencia de potencial eléctrico generado por el sistema termoeléctrico se considera que la variación del coeficiente de Seebeck es muy poca, de tal manera que se puede considerar constante, esto es:

ROJAS-AVILA, Apolo, VALLE-HERNÁNDEZ, Julio, CASTILLOJIMÉNEZ, Víctor, GALLARDO-VILLAREAL, José Manuel y ALONSOALFARO Jorge G. Análisis energético de un sistema termoeléctrico para el aprovechamiento del calor residual en un motor Turbofan mediante dinámica de fluidos computacional. Revista de Sistemas Experimentales. 2019 
$V_{\alpha}=s\left(T_{h}-T_{c}\right)$

La transferencia de calor en el termoeléctrico parte de la temperatura en la cara caliente $\left(T_{h}\right)$ del semiconductor y del flujo de calor en la cara fría $\left(\mathrm{T}_{\mathrm{c}}\right)$, como se muestra en la figura 2. El flujo de calor, en Tc, está en función de la transferencia de calor por convección forzada debido al flujo secundario del motor.

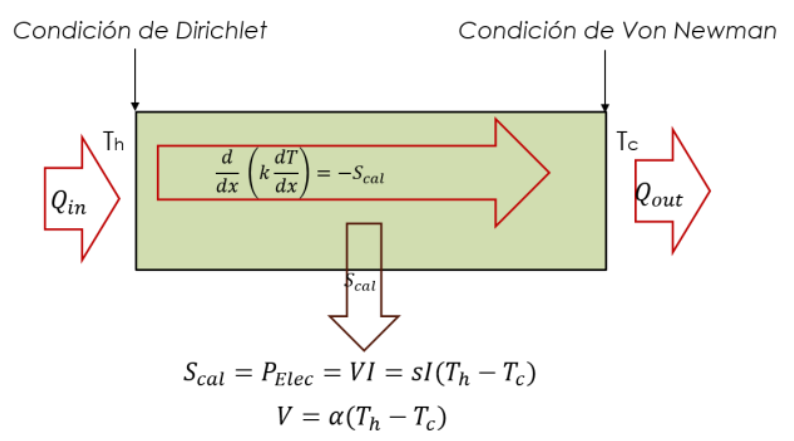

Figura 2 Transferencia de calor en un segmento termoeléctrico de los que se compone cada módulo termoeléctrico

Para el cálculo de la potencia eléctrica máxima generada por el sistema termoeléctrico se utiliza la ecuación 3.

$P_{E l}=I^{2} * R_{T} * n$

Donde:

$\mathrm{P}_{\mathrm{El}}$ : Potencia eléctrica

I: Corriente eléctrica

$\mathrm{R}_{\mathrm{T}}$ : Resistencia eléctrica en el módulo

n: número de segmentos semiconductores

El cálculo de la corriente eléctrica se determina para los parámetros máximos como el voltaje de Seebeck entre dos veces la resistencia eléctrica del material semiconductor. En el anexo 2 se muestran los valores del material semiconductor.

Para realizar un balance de energía, sobre un elemento termoeléctrico, se parte de la ecuación general de conducción de calor. Considerando que el estudio se realiza en estado estacionario, con conductividad y área de transferencia de calor constante, la ecuación de conducción de calor queda como:

$$
\left(\frac{\partial^{2} T}{\partial x^{2}}\right)+\left(\frac{\partial^{2} T}{\partial y^{2}}\right)+\left(\frac{\partial^{2} T}{\partial z^{2}}\right)-\frac{\dot{s}_{C a l}}{k}=0
$$

Donde $S_{\text {cal }}$ se considera el sumidero de calor equivalente a la energía eléctrica generada por el efecto termoeléctrico.

Una vez determinada la potencia generada por parte del sistema termoeléctrico, se realiza el análisis energético para determinar la viabilidad energética de la implementación del sistema, y obtener la eficiencia de cogeneración.

A partir de la ecuación 4 se realiza la discretización para la transferencia de calor de la condición de Dirichlet a la de Von Newman, obteniendo las ecuaciones 5, para la transferencia de calor en la condición de frontera donde se conoce la temperatura y a través del segmento termoeléctrico, y 6 para la temperatura en la condición de Dirichlet ya que se conoce el flujo de calor en función a la convección forzada que se genera en el borde.

$a_{p} T_{p}=a_{E} T_{E}+a_{W} T_{w}+b$

Donde:

$$
\begin{aligned}
& a_{E}=\frac{k_{j+1}}{\left(\delta x_{i}\right)_{j+1}} \\
& a_{W}=\frac{k_{j-1}}{\left(\delta x_{i}\right)_{j-1}} \\
& a_{p}=a_{E}+a_{W} \\
& b=\dot{S}_{C a l} \Delta x_{i}
\end{aligned}
$$

En la figura 3 se puede observar los incrementos mostrados en la ecuación 5, para la discretización de la transferencia de calor en un segmento termoeléctrico.

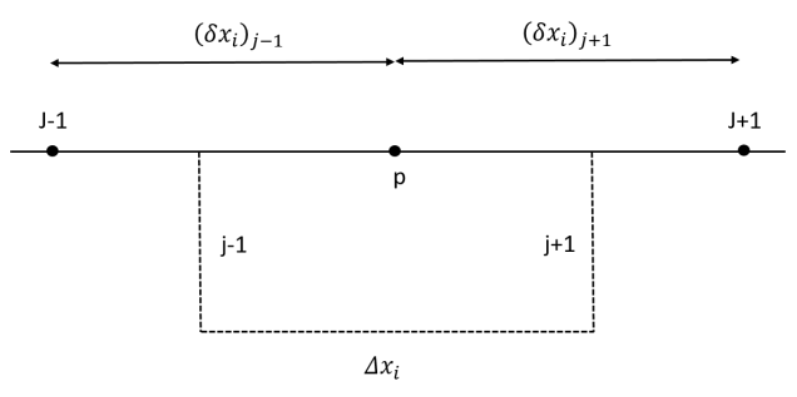

Figura 3 Discretización de cada punto de la malla para un segmento termoeléctrico

$$
a_{J-1} T_{J-1}=a_{J} T_{J}+b
$$

Donde:

$a_{J}=\frac{\frac{k_{j-1}}{2}}{(\delta x)_{\frac{j-1}{2}}}$

ROJAS-AVILA, Apolo, VALLE-HERNÁNDEZ, Julio, CASTILLOJIMÉNEZ, Víctor, GALLARDO-VILLAREAL, José Manuel y ALONSOALFARO Jorge G. Análisis energético de un sistema termoeléctrico para el aprovechamiento del calor residual en un motor Turbofan mediante dinámica de fluidos computacional. Revista de Sistemas Experimentales. 2019 


$$
\begin{aligned}
& b=S_{0} \Delta x+q_{J-1} \\
& a_{J-1}=a_{J}-S_{1} \Delta x
\end{aligned}
$$

En el borde en el cual se conoce el flujo de calor (ecuación 6) se discretiza tomando en cuenta los incrementos mostrados en la figura 4.

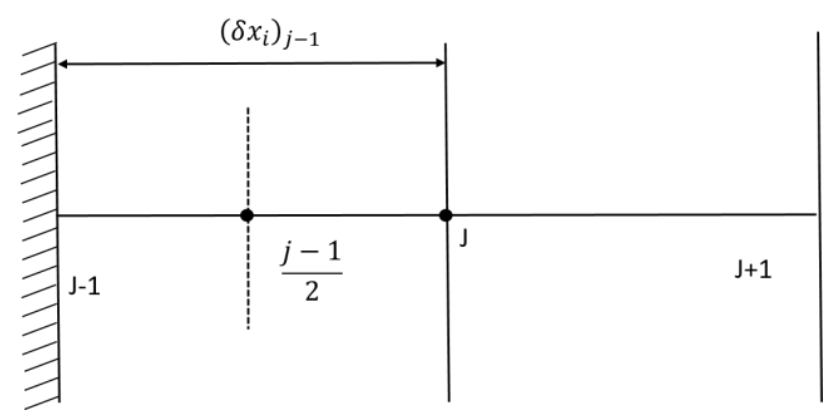

Figura 4 Incrementos para la discretización en el borde con condición de Dirichlet

\section{Resultados del modelo}

Los resultados obtenidos se dividen en:

A.-Parámetros de operación del motor turbofan. B.-Distribución de temperaturas de la sección de interés del motor.

C.- Gradiente de temperaturas y potencial eléctrico en un TEG.

D.- Potencia eléctrica máxima obtenida por cada TEG.

A. Parámetros de operación del motor turbofan.

Mediante las características conocidas el motor Trent 900 se realizó el análisis termodinámico en condiciones de vuelo de crucero para determinar la temperatura de estancamiento $\left(\mathrm{T}_{\mathrm{T}}\right)$, presión de estancamiento $\left(\mathrm{P}_{\mathrm{T}}\right)$, la temperatura estática y el trabajo realizado $(\mathrm{W})$.

\begin{tabular}{|l|r|r|r|r|}
\hline Punto & \multicolumn{1}{c|}{$\mathbf{T}_{\mathbf{T}}[\mathbf{K}]$} & $\mathbf{P}_{\mathbf{T}}[\mathbf{K} \mathbf{p a}]$ & $\mathbf{T}[\mathbf{K}]$ & $\mathbf{w}[\mathbf{K J} / \mathbf{K g}]$ \\
\hline 0 & 250.3116 & 36.05 & 220 & 0 \\
\hline 1 & 250.3116 & 35.019 & 220 & 0 \\
\hline 2 & 776.03 & 1364.922 & 621.99 & -432 \\
\hline 3 & 1800 & 1337.62 & 1800 & -528 \\
\hline 4 & 987.545 & 119.0062 & 670 & 960 \\
\hline 5 & 987.545 & ---------- & 670 & 0 \\
\hline 6 & 301.487 & 64.654 & 222.45 & 0 \\
\hline 7 & 301.487 & ---------- & 222.45 & 0 \\
\hline
\end{tabular}

Tabla 2 condiciones de temperatura, presión, calor y trabajo en cada punto del ciclo de propulsión del motor Trent 900
B. Distribución de temperaturas de la sección de interés del motor

A partir del modelo CFD realizado con las características y parámetros determinados del motor turbofan (Tabla 2) se obtuvo como resultado la distribución de temperaturas en la sección de interés (Gráfico 1). La distribución de temperatura obtenida se utiliza como la temperatura de la cara caliente en el segmento termoeléctrico descrito en la sección anterior (modelado del sistema termoeléctrico) como condición de Dirichlet.

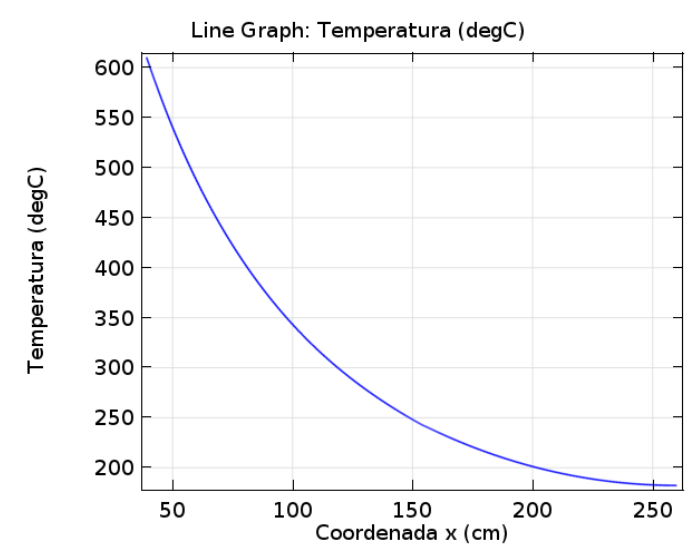

Gráfico 1 Distribución de temperatura en la cara externa de la sección que recubre la turbina y en la tobera

\section{Gradiente de temperaturas y potencial eléctrico en un TEG}

Mediante la distribución de temperatura se realizó un modelo del comportamiento de un módulo termoeléctrico bajo condiciones de temperatura en el rango de la gráfica 1, obteniendo como resultado diferencias de temperatura de alrededor de $100{ }^{\circ} \mathrm{C}$ y potenciales eléctricos de $0.022 \mathrm{~V}$ por cada segmento termoeléctrico. En la figura 5 se ilustra el segmento termoeléctrico en el TEG modelado en CFD, el cual tiene una dimensión de $1 \times 0.8$ $\mathrm{cm}$.

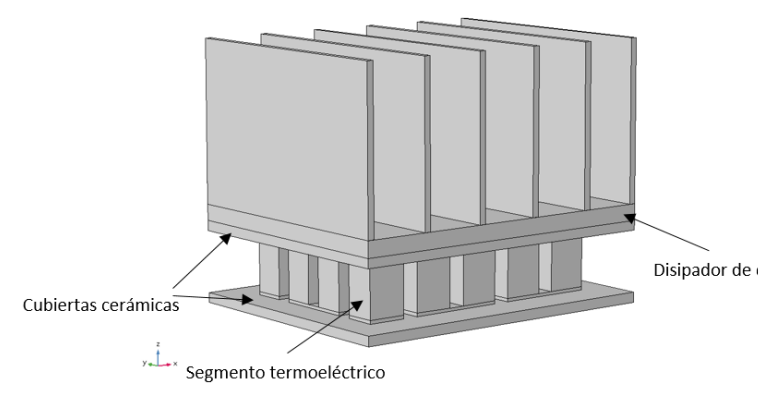

Figura 5 Modulo termoeléctrico con disipador de calor

ROJAS-AVILA, Apolo, VALLE-HERNÁNDEZ, Julio, CASTILLOJIMÉNEZ, Víctor, GALLARDO-VILLAREAL, José Manuel y ALONSOALFARO Jorge G. Análisis energético de un sistema termoeléctrico para el aprovechamiento del calor residual en un motor Turbofan mediante dinámica de fluidos computacional. Revista de Sistemas Experimentales. 2019 
En el grafico 2 se muestra la distribución de temperaturas a través de las caras de un elemento termoeléctrico.

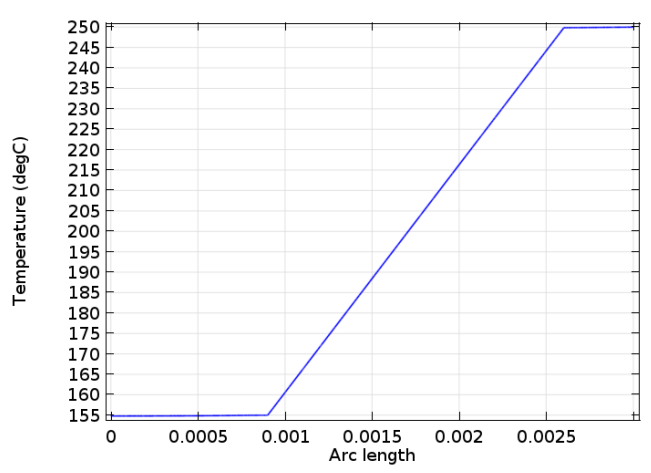

Gráfico 2 Distribución de temperaturas en cada segmento termoeléctrico

El potencial eléctrico generado se debe a la diferencia de temperaturas en cada segmento termoeléctrico. El gráfico 3 muestra la generación de potencial eléctrico en un segmento que compone al módulo.

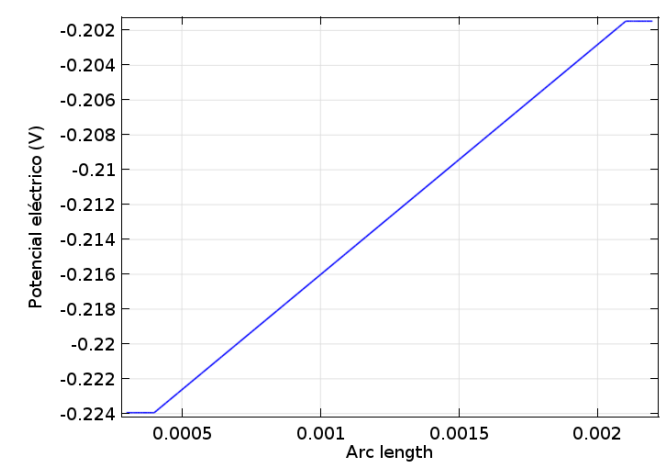

Gráfico 3 Potencial eléctrico en un segmento termoeléctrico

D. Potencia eléctrica generada por cada TEG

A partir de los resultados obtenidos se puede determinar la cantidad de energía generada por cada módulo termoeléctrico obteniendo $8.7 \mathrm{~W}$ eléctricos por cada $16 \mathrm{~cm}^{2}$.

\section{Análisis de resultados}

Para obtener el gradiente de temperaturas se utiliza un disipador de calor ya que se mejora considerable mente el gradiente de temperaturas, de $40{ }^{\circ} \mathrm{C}$ a $100^{\circ} \mathrm{C}$.

A partir de los resultados obtenidos se estima la generación de alrededor de $5.4 \mathrm{KW} / \mathrm{m}^{2}$.
La energía eléctrica generada puede ser aprovechada en sistemas de la aeronave tales como la iluminación en secciones de pasajeros ya que el consumo de potencia es de alrededor de $5.6 \mathrm{KW}$.

\section{Discusión de resultados}

Las potenciales aplicaciones, de la generación de energía termoeléctrica, en la aeronave son principalmente en sistemas secundarios de la aeronave tales como entretenimiento, carga de dispositivos móviles, iluminación, etc.

No obstante, que los resultados obtenidos son teóricos, por lo que deberán ser validados en trabajos futuros, los rangos de potencia obtenidos se muestran prometedores para su implementación como sistema de cogeneración.

\section{Agradecimiento}

Agradecimiento a CONACYT por el apoyo económico a través del programa de becas de posgrado para carreras en PNPC.

\section{Referencias}

[1] Fraisse, G., Ramousse, J., Sgorlon, D., \& Goupil, C. (2013). Comparison of different modeling approaches for thermoelectric elements. Energy Conversion and Management, $65,351-356$.

[2] Chen, J., Yan, Z., \& Wu, L. (1996). The influence of Thomson effect on the maximum power output and maximum efficiency of a thermoelectric generator. Journal of Applied Physics, 79(11), 8823-8828.

[3] Navarro, P. M. (2017). Análisis por elementos finitos de los generadores termoeléctricos y sus aplicaciones aeroespaciales (Tesis de doctorado). Universidad Politécnica de Valencia, España.

[4] Temizer, I., \& Ilkiliç, C. (2016). The performance and analysis of the thermoelectric generator system used in diesel engines. Renewable and Sustainable Energy Reviews, 63, $141-151$. 
[5] Kousksou, T., Bédécarrats, J. P., Champier, D., Pignolet, P., \& Brillet, C. (2011). Numerical study of thermoelectric power generation for a helicopter conical nozzle. Journal of Power Sources, 196(8), 4026-4032.

[6] Champier, D. (2017). Thermoelectric generators: A review of applications. Energy Conversion and Management, 140, 167-181.

[7] Janak, L., Ancik, Z., Vetiska, J., \& Hadas, Z. (2015). Thermoelectric Generator Based on MEMS Module as an Electric Power Backup in Aerospace Applications. Materials Today: Proceedings, 2(2), 865-870.

[8] Samson, D., Kluge, M., Becker, T., \& Schmid, U. (2011). Wireless sensor node powered by aircraft specific thermoelectric energy harvesting. Sensors and Actuators, A: Physical, 172(1), 240-244.

[9] Hernández, V.-, Alejandro, O., \& RojasAvila, R. (2018). Análisis energético de un sistema de generación termoeléctrica utilizando calor residual de celdas de combustible. Revista de Ingeniería Mecánica, 2(6), 20-27.

[10] Undurraga Almaraz, A. (2014). Viabilidad de los materiales termoeléctricos (tesis de maestría). Universidad de Barcelona, España.

[11] Y. Niño. (2012) Método de los volúmenes finitos. Universidad de Chile, departamento de ingeniería civil.

\section{Anexos}

\section{Anexo 1}

Características y eficiencias usadas para el calculo de las propiedades termodinámicas del ciclo de propulsión de un motor Trent 900, tipo turbofan.

\begin{tabular}{|l|r|}
\hline$\Pi_{\text {Fan }}$ & 1.5 \\
\hline$\eta_{N}$ & $97 \%$ \\
\hline$\eta_{D}$ & $99 \%$ \\
\hline$\eta_{\text {ise }}$ & $87 \%$ \\
\hline$\eta_{\text {isf }}$ & $89 \%$ \\
\hline$\eta_{\text {comb }}$ & $98 \%$ \\
\hline$\Delta \mathrm{P}_{\text {com }}$ & $2 \%$ \\
\hline $\mathrm{K}$ & 1.4 \\
\hline$C_{p}($ Aire $)$ & $1.005\left[\mathrm{KJ} / \mathrm{Kg}^{*} \mathrm{~K}\right]$ \\
\hline$C_{p g}$ (Gases de escape) & $1.147[\mathrm{KJ} / \mathrm{Kg} * \mathrm{~K}]$ \\
\hline $\mathrm{K}_{\mathrm{g}}$ (Gases de escape) & 1.33 \\
\hline
\end{tabular}

\section{Anexo 2}

Propiedades térmicas y eléctricas del telururo de bismuto.

\begin{tabular}{|c|r|}
\hline Propiedad & \multicolumn{1}{c|}{ Valor } \\
\hline Coeficiente de Seebeck $(\mathrm{s})$ & $200 \frac{\mu \mathrm{V}}{\mathrm{K}}$ \\
\hline Conductividad térmica $(\mathrm{k})$ & $1.8 \frac{\mathrm{W}}{\mathrm{mK}}$ \\
\hline Resistividad eléctrica $(\rho)$ & $1.5 \times 10^{-5} \Omega * \mathrm{~m}$ \\
\hline
\end{tabular}

